\title{
Passive Sampling and Dosing of Aquatic Organic Contaminant Mixtures for Ecotoxicological ANALySES.
}

Kilian E.C. Smith ${ }^{a, b, *}$ and Yoonah Jeonga,c.

a Environmental Safety Group, KIST Europe, Korea Institute of Science and Technology, Campus E 7.1, Saarbrücken, Germany.

b Current address: Department of Water, Environment, Construction and Safety, University of Applied Sciences Magdeburg-Stendal, Magdeburg, Germany.

c Current address: Department of Land, Water and Environment Research, Korea Institute of Civil Engineering and Building Technology (KICT), Gyeonggi-Do, Republic of Korea.

* Corresponding author. e-mail: kilian.smith@h2.de. Tel. +49 (0)391 8864921. 
Pages S4 to S7. Full details of experiments for determining the ecotoxicity of PAH mixtures in the laboratory.

Table S1. List of the 44 target compounds analysed in the study along with their physicochemical properties and typical application.

Table S2. Passive sampler uptake rate constants $(1 / \mathrm{h})$ for the different PAHs in the set-ups with and without dissolved organic carbon (DOC).

Table S3. Equilibrium passive sampler concentrations $\left(C_{P D M S}\right.$,equil., $\left.\mu \mathrm{g} / \mathrm{L}\right)$ from fitting of the uptake curves together with the calculated dissolved concentrations $\left(C_{\text {free,calculated }} \mu \mathrm{g} / \mathrm{L}\right)$ for the set-ups with and without dissolved organic carbon (DOC).

Table S4. Mean ( $n=2)$ passive sampler concentrations (ng/kg PDMS) for the WWTP inflow and outflow samples, as well as the receiving Saar river.

Figure S1. Schematic of the experimental set-up.

Figure S2. Photographs of the PDMS silicone sheets used as passive samplers recovered from the WWTP inflow $(A)$ and outflow $(B)$ after a 10-day deployment in September.

Figure S3. Measured aqueous concentrations ( $\mu \mathrm{g} / \mathrm{L})$ of acenaphthene, phenanthrene, fluoranthene and benzo(a)pyrene in the set-ups containing medium with and without $100 \mathrm{mg} / \mathrm{L}$ dissolved organic carbon (DOC).

Figure S4. Comparison of the passive sampler concentrations from each set-up determined directly after sampling or after application as passive dosers in the Microtox bioassay.

Figure S5. Measured passive sampler concentrations $(\mu \mathrm{g} / \mathrm{L})$ of acenaphthene, phenanthrene, fluoranthene and benzo(a)pyrene in the set-ups containing medium with and without $100 \mathrm{mg} / \mathrm{L}$ dissolved organic carbon (DOC).

Figure S6. PAH chemical activity profiles for samples taken at 2, 13 and $96 \mathrm{~h}$ at the start of the Microtox bioassay for aqueous sample extraction and spiking, passive sampler extraction and spiking and passive sampling and dosing.

Figure S7. PAH mixture toxicities after extraction of $5 \mathrm{~mL}$ saline medium or saline medium plus DOC, exchange into $0.05 \mathrm{~mL}$ DMSO and spiking $1 \mu \mathrm{L}$ of this extract into the Microtox bioassay.

Figure S8. PAH mixture toxicities after extraction of passive samplers deployed for increasing lengths of time in $1 \mathrm{~mL}$ hexane, exchanging into $0.05 \mathrm{~mL}$ DMSO and spiking $1 \mu \mathrm{L}$ of this extract into the Microtox bioassay.

Figure S9. Ratio of the mean concentrations measured in the thin $(76 \mu \mathrm{m})$ and thick $(203 \mu \mathrm{m})$ passive samplers deployed in the WWTP inflow and outflow as well as Saar river. 
Figure S10. Initial mixture profiles of the environmental mixtures in the toxicity bioassays as set by equilibrium passive sampling and dosing (left column) and sampler extraction and spiking (right panel).

Figure S11. Concentration-response curve for the reference compounds $17 \beta$ estradiol in the ER Calux bioassay.

Figure S12. Calux bioassay concentration-response curve for the different levels of the spiked extracts (A) and passively dosed samples (B) from the inflow passive samplers recovered after the $3^{\text {rd }}$ deployment. 


\section{Ecotoxicity of PAH mixtures in the laboratory}

A schematic of the set-up is shown in Figure S1 below. Amber Duran Schott ${ }^{\circledR}$ bottles were filled with $2.1 \mathrm{~L}$ of saline medium ( $2 \%$ sodium chloride solution for compatibility with the Microtox ${ }^{\circledR}$ test). One bottle contained medium only, whereas the other contained dissolved organic carbon (DOC) at $100 \mathrm{mg} / \mathrm{L}$ as a sorbing phase. Here, humic acid as a model DOC was directly dissolved in the medium and neutralized to pH 7 using sodium hydroxide.

Passive dosing for producing identical dissolved concentrations. PAH dissolved concentrations in both set-ups were set at the same levels by passive dosing from PDMS silicone sheets. PDMS sheets (each 6 × $15 \mathrm{~cm}$ and $203 \mu \mathrm{m}$ thick) were cleaned by three overnight soakings in methanol, dried and loaded by non-depletion equilibrium partitioning for 24 hours from $300 \mathrm{~mL}$ of a methanol solution containing acenaphthene at $1.620 \mathrm{mg} / \mathrm{mL}$, phenanthrene at $0.305 \mathrm{mg} / \mathrm{mL}$, fluoranthene at 5.357 $\mathrm{mg} / \mathrm{mL}$ and benzo(a)pyrene at $0.107 \mathrm{mg} / \mathrm{mL}$. These correspond to $10 \%, 2 \%, 50 \%$ and $12 \%$ of the methanol saturation values of each of the PAHs (values taken from Reichenberg et al, 2008). ${ }^{1}$ The PDMS and dissolved PAH concentrations were thus also $10 \%, 2 \%, 50 \%$ and $12 \%$ of the respective saturation concentrations. These were selected so that the mixture toxicity measured in the Microtox test was approximately $50 \%$ of the maximum luminescence inhibition to allow differences in the mixture toxicity to be seen. After loading, the sheets were rinsed three times with MilliQ water to remove residual methanol, before sandwiching in sets of two between stainless steel mesh holders held together using stainless steel crocodile clips. Three such mesh holders were placed in each bottle, giving a total dosing phase volume of 10.96 $\mathrm{cm}^{3}$ and surface area of $540 \mathrm{~cm}^{2}$ per set-up. 
Passive sampling. As passive samplers, $1 \mathrm{~cm}$ diameter disks were punched out of the $76 \mu \mathrm{m}$ thick PDMS sheet (mean mass $11.6 \mathrm{mg}, \mathrm{CV} 3.9 \%, \mathrm{n}=25$ ) using a stainless steel punch and cleaned as above. Passive sampler disks $(n=36)$ were sandwiched in a stainless steel mesh to give a total passive sampler volume of $0.21 \mathrm{~cm}^{3}$ and surface area of $56.55 \mathrm{~cm}^{2}$. This corresponds to $2 \%$ and $10 \%$ of the passive dosing phase volume and surface area, respectively. This avoided depletion of the passive dosing sheets and ensured dissolved concentrations were determined by equilibrium partitioning.

Passive dosing of the PAHs was started by adding three mesh holders each containing the paired passive dosing sheets to each set-up. These were stirred overnight to allow a partitioning equilibrium to be reached, and passive sampling started by adding a mesh holder containing the 36 passive sampler disks to each set-up. Various sample types were then collected for chemical analyses and toxicity testing as described below.

Samples for toxicity testing and chemical analyses. To determine the background toxicity and blank levels, 6 passive sampler disks were added to each set-up prior to initiating passive dosing of the PAHs. These were exposed for 24 hours before recovering and storing separately in HPLC vials. In parallel, three $5 \mathrm{~mL}$ aqueous samples were taken from each bottle and stored separately in $20 \mathrm{~mL}$ headspace vials.

Aqueous samples with a volume of $5 \mathrm{~mL}$ were collected at $0,13,56,152$ and 175 hours ( $n=3$ each). These were extracted by shaking twice on a horizontal shaker at $175 \mathrm{rpm}$ with $1 \mathrm{~mL}$ hexane for two hours each time, the extracts combined and blown to incipient dryness under nitrogen before taking up in $0.5 \mathrm{~mL}$ methanol for analysis. Passive samplers for direct determination of their concentrations were removed at 56 , 
96 and 152 hours ( $n=3$ each time). However, depletion of the passive sampler disks after application in passive dosing mode was negligible (see main text and Figure S4), and their concentrations were also measured after using them for the toxicity testing. Therefore, in total passive sampler concentrations were determined at $0,2,4,8,13$, 28, 56, 96 and $152 \mathrm{~h}(\mathrm{n}=3$ each time except for $56 \mathrm{~h}$ where a total of 6 disks were available, i.e., 3 after passive dosing and 3 for direct analysis). The passive sampler disks were directly extracted in the HPLC vials used to store them by adding $1 \mathrm{~mL}$ methanol for a minimum of 24 hours before analysis. This methanol volume was sufficient such that less than $0.4 \%$ of the PAHs remained in the silicone at the end of the extraction step.

Samples for toxicity testing. Three types of samples were collected for the mixture toxicity testing in the Microtox bioassay: aqueous samples for extraction and spiking, passive samplers for extraction and spiking and finally passive samplers for direct application as equilibrium passive dosers.

Five $\mathrm{mL}$ aqueous samples were collected at 4 and $56 \mathrm{~h}(\mathrm{n}=3$ each time). These were extracted as described above, before exchanging into $0.05 \mathrm{~mL}$ DMSO for spiking into the toxicity bioassay.

Passive samplers were collected at 4, 13 and $56 \mathrm{~h}$ for testing the sampler extraction and spiking approach ( $\mathrm{n}=3$ each time). These were extracted overnight in $1 \mathrm{~mL}$ hexane before exchanging into $0.05 \mathrm{~mL}$ DMSO for spiking into the toxicity bioassay.

Finally, passive sampler disks for testing in passive dosing mode were collected at 0 (i.e., the blank disks from above), 2, 4, 8, 13, 28, 56 and $96 \mathrm{~h}$ ( $\mathrm{n}=3$ each time). These were stored in HPLC vials until direct application as passive dosers in the toxicity bioassay. 
Recovery of the aqueous sample extraction method. An initial recovery test was performed to quantify the losses of the extraction and blow-down steps during analysis of the aqueous samples. For this, $100 \mathrm{ng}$ each of acenaphthene, phenanthrene, fluoranthene and benzo(a)pyrene were added to $5 \mathrm{~mL}$ of $2 \%$ saline medium $(n=3)$. These were extracted as described above and analysed by HPLC-DAD. Mean recoveries from the extraction test were $75.3 \%$ (coefficient of variation (CV) $2.0 \%$ ) for acenaphthene, $82.7 \%$ (CV 0.6\%) for phenanthrene, $87.3 \%$ (CV 4.6\%) for fluoranthene and $93.9 \%$ (CV 2.8\%) for benzo(a)pyrene. These were used to correct the concentrations measured in the aqueous samples.

Data handling. Time-concentration curves describing uptake by the passive sampling disks from constant dissolved concentration were fitted with a one-compartment model (Equation S1), where $C_{P M D S(t)}(\mu \mathrm{g} / \mathrm{L})$ is the water concentration at time $t(\mathrm{~h}), C_{P D M S(e q)}$ $(\mu \mathrm{g} / \mathrm{L})$ is the equilibrium water concentration, and $k_{\text {uptake }}(1 / \mathrm{h})$ is the uptake rate constant.

$C_{P D M S(t)}=C_{P D M S(e q)}\left(1-\exp ^{-k_{\text {uptake }} t}\right) \quad$ Equation S1

As a quantitative measure of the time to reach equilibrium, t95\% (h) the time to reach 95\% steady state was used (Equation S2).

$t_{95 \%}=\frac{3}{k_{\text {uptake }}}$

Equation S2

Data were fitted using the least-squares method with Graphpad Prizm 5 (San Diego, CA). 
Table S1. List of the 44 target compounds analysed in the study along with their physicochemical properties and typical application. Log Kow and water solubility (mg/L) were calculated using KOWWIN v1.68 and WSKOW v1.41 in EPI Suite (USEPA 2012). Log D at pH 7 was obtained from Marvin sketch 6.2.2 (ChemAxon 2014). Compound names in bold are priority substances in the EU WFD (2013/39/EC).

\begin{tabular}{|c|c|c|c|c|c|c|c|}
\hline Chemical & CAS number & Mass & Formula & $\log \mathrm{K}_{\mathrm{ow}}$ & $\log \mathrm{D}$ at $\mathrm{pH} 7$ & $\begin{array}{l}\text { Water solubility } \\
\text { at } 25^{\circ} \mathrm{C}(\mathrm{mg} / \mathrm{L})\end{array}$ & Use \\
\hline $2,4 \mathrm{D}$ & $94-75-7$ & 221.04 & $\mathrm{C}_{8} \mathrm{H}_{6} \mathrm{Cl}_{2} \mathrm{O}_{3}$ & 2.62 & -0.94 & 336.20 & Herbicide \\
\hline Alachlor & $15972-60-8$ & 269.77 & $\mathrm{C}_{14} \mathrm{H}_{20} \mathrm{CINO}_{2}$ & 3.37 & 3.59 & 18.07 & Herbicide \\
\hline Atenolol & $29122-68-7$ & 266.34 & $\mathrm{C}_{14} \mathrm{H}_{22} \mathrm{~N}_{2} \mathrm{O}_{3}$ & -0.03 & -2.14 & 685.20 & Pharmaceutical \\
\hline Atrazine & $1912-24-9$ & 215.68 & $\mathrm{C}_{8} \mathrm{H}_{14} \mathrm{CIN}_{5}$ & 2.82 & 2.20 & 214.10 & Herbicide \\
\hline Bezafibrate & $41859-67-0$ & 416.13 & $\mathrm{C}_{18} \mathrm{H}_{14} \mathrm{Cl}_{4} \mathrm{~N}_{2} \mathrm{O}$ & 4.25 & 0.97 & 1.22 & Pharmaceutical \\
\hline Bifenox & $42576-02-3$ & 342.13 & $\mathrm{C}_{14} \mathrm{H}_{9 \mathrm{Cl}_{2} \mathrm{NO}_{5}}$ & 4.15 & 4.63 & 0.42 & Herbicide \\
\hline Carbamazepine & $298-46-4$ & 236.27 & $\mathrm{C}_{15} \mathrm{H}_{12} \mathrm{~N}_{2} \mathrm{O}$ & 2.25 & 2.77 & 17.66 & Pharmaceutical \\
\hline Carbendazim & 10605-21-7 & 191.19 & $\mathrm{C}_{9} \mathrm{H}_{9} \mathrm{~N}_{3} \mathrm{O}_{2}$ & 1.55 & 1.80 & 2441.00 & Fungicide \\
\hline Chlorpyrifos & $2921-88-2$ & 350.59 & $\mathrm{C}_{9} \mathrm{H}_{11} \mathrm{Cl}_{3} \mathrm{NO}_{3} \mathrm{PS}$ & 4.66 & 4.78 & 0.36 & Insecticide \\
\hline Clopyralid & $1702-17-6$ & 192.00 & $\mathrm{C}_{6} \mathrm{H}_{3} \mathrm{Cl}_{2} \mathrm{NO}_{2}$ & 1.63 & -1.11 & 5974.00 & Herbicide \\
\hline Clotrimazole & $23593-75-1$ & 344.84 & $\mathrm{C}_{22} \mathrm{H}_{17} \mathrm{ClN}_{2}$ & 6.26 & 5.73 & 0.03 & Fungicide \\
\hline Cyanazine & $21725-46-2$ & 240.69 & $\mathrm{C}_{9} \mathrm{H}_{13} \mathrm{CIN}_{6}$ & 2.51 & 1.96 & 183.80 & Herbicide \\
\hline
\end{tabular}




\begin{tabular}{|c|c|c|c|c|c|c|c|}
\hline Cybutryne (Irgarol) & $28159-98-0$ & 253.37 & $\mathrm{C}_{11} \mathrm{H}_{19} \mathrm{~N}_{5} \mathrm{~S}$ & 4.07 & 2.97 & 7.52 & Algicide \\
\hline DEHP & $117-81-7$ & 390.56 & $\mathrm{C}_{24} \mathrm{H}_{38} \mathrm{O}_{4}$ & 8.39 & 8.03 & 0.00 & Industrial \\
\hline Dichlorvos & $62-73-7$ & 220.98 & $\mathrm{C}_{4} \mathrm{H}_{7} \mathrm{Cl}_{2} \mathrm{O}_{4} \mathrm{P}$ & 0.60 & 1.37 & 1889.00 & Insecticide \\
\hline Diclofenac & $15307-79-6$ & 296.15 & $\mathrm{C}_{14} \mathrm{H}_{11} \mathrm{Cl}_{2} \mathrm{NO}_{2}$ & 4.02 & 1.37 & 4.52 & Pharmaceutical \\
\hline Diuron & $330-54-1$ & 232.02 & $\mathrm{C}_{9} \mathrm{H}_{10} \mathrm{Cl}_{2} \mathrm{~N}_{2} \mathrm{O}$ & 2.67 & 2.53 & 150.60 & Herbicide \\
\hline Econazole & $27220-47-9$ & 381.68 & $\mathrm{C}_{18} \mathrm{H}_{15} \mathrm{Cl}_{3} \mathrm{~N}_{2} \mathrm{O}$ & 5.61 & 5.21 & 0.06 & Fungicide \\
\hline Fenamidone & $161326-34-7$ & 311.40 & $\mathrm{C}_{17} \mathrm{H}_{17} \mathrm{~N}_{3} \mathrm{OS}$ & 3.45 & 4.74 & 11.95 & Fungicide \\
\hline Flufenacetat & $142459-58-3$ & 363.33 & $\mathrm{C}_{14} \mathrm{H}_{13} \mathrm{~F}_{4} \mathrm{~N}_{3} \mathrm{O}_{2} \mathrm{~S}$ & 2.39 & 3.22 & 9.52 & Herbicide \\
\hline Fluroxypyr & $69377-81-7$ & 255.03 & $\mathrm{C}_{7} \mathrm{H}_{5} \mathrm{Cl}_{2} \mathrm{FN}_{2} \mathrm{O}_{3}$ & 1.17 & -1.91 & 5551.00 & Herbicide \\
\hline Furalaxyl & $57646-30-7$ & 301.34 & $\mathrm{C}_{17} \mathrm{H}_{19} \mathrm{NO}_{4}$ & 2.70 & 3.21 & 71.18 & Fungicide \\
\hline Gemfibrozil & $25812-30-0$ & 250.33 & $\mathrm{C}_{15} \mathrm{H}_{22} \mathrm{O}_{3}$ & 4.77 & 1.85 & 4.96 & Pharmaceutical \\
\hline Ibuprofen & $15687-27-1$ & 206.28 & $\mathrm{C}_{13} \mathrm{H}_{18} \mathrm{O}_{2}$ & 3.79 & 1.71 & 41.05 & Pharmaceutical \\
\hline Imazapic & $104098-48-8$ & 275.30 & $\mathrm{C}_{14} \mathrm{H}_{17} \mathrm{~N}_{3} \mathrm{O}_{3}$ & 2.11 & -1.16 & 132.50 & Herbicide \\
\hline Isoproturon & $34123-59-6$ & 206.28 & $\mathrm{C}_{12} \mathrm{H}_{18} \mathrm{~N}_{2} \mathrm{O}$ & 2.84 & 2.57 & 143.80 & Herbicide \\
\hline Ketoconazole & $65277-42-1$ & 531.43 & $\mathrm{C}_{26} \mathrm{H}_{28} \mathrm{Cl}_{2} \mathrm{~N}_{4} \mathrm{O}_{4}$ & 4.45 & 4.06 & 0.09 & Fungicide \\
\hline MCPA & $94-74-6$ & 200.62 & $\mathrm{C}_{9} \mathrm{H}_{9} \mathrm{ClO}_{3}$ & 2.52 & -0.87 & 180.90 & Herbicide \\
\hline Metalaxyl & $57837-19-1$ & 279.33 & $\mathrm{C}_{15} \mathrm{H}_{21} \mathrm{NO}_{4}$ & 1.70 & 2.12 & 560.00 & Fungicide \\
\hline Metoprolol & $51384-51-1$ & 267.36 & $\mathrm{C}_{15} \mathrm{H}_{25} \mathrm{NO}_{3}$ & 1.69 & -0.81 & 4777.00 & Pharmaceutical \\
\hline Miconazole & $22916-47-8$ & 416.13 & $\mathrm{C}_{18} \mathrm{H}_{14} \mathrm{Cl}_{4} \mathrm{~N}_{2} \mathrm{O}$ & 6.25 & 5.82 & 0.01 & Fungicide \\
\hline
\end{tabular}




\begin{tabular}{|c|c|c|c|c|c|c|c|}
\hline Orysastrobin & $248593-16-0$ & 391.42 & $\mathrm{C}_{18} \mathrm{H}_{25} \mathrm{~N}_{5} \mathrm{O}_{5}$ & 2.77 & 2.89 & 14.79 & Fungicide \\
\hline Oxadixyl & $77732-09-3$ & 278.30 & $\mathrm{C}_{14} \mathrm{H}_{18} \mathrm{~N}_{2} \mathrm{O}_{4}$ & 1.40 & 1.79 & 3398.00 & Fungicide \\
\hline Pentachlorophenol & $87-86-5$ & 266.34 & $\mathrm{C}_{6} \mathrm{HCl}_{5} \mathrm{O}$ & 4.74 & 2.95 & 3.09 & Insecticide \\
\hline Quinoxyfen & $124495-18-7$ & 308.14 & $\mathrm{C}_{15} \mathrm{H}_{8} \mathrm{Cl}_{2} \mathrm{FNO}$ & 5.69 & 4.98 & 1.15 & Fungicide \\
\hline Simazine & $122-34-9$ & 201.66 & $\mathrm{C}_{7} \mathrm{H}_{12} \mathrm{ClN}_{5}$ & 2.40 & 1.78 & 589.90 & Herbicide \\
\hline Sulfamethoxazole & $723-46-6$ & 253.28 & $\mathrm{C}_{10} \mathrm{H}_{11} \mathrm{~N}_{3} \mathrm{O}_{3} \mathrm{~S}$ & 0.48 & 0.14 & 3942.00 & Pharmaceutical \\
\hline Terbutryn & $886-50-0$ & 241.36 & $\mathrm{C}_{10} \mathrm{H}_{19} \mathrm{~N}_{5} \mathrm{~S}$ & 3.77 & 2.85 & 16.89 & Algicide \\
\hline Thiabendazole & $148-79-8$ & 201.25 & $\mathrm{C}_{10} \mathrm{H}_{7} \mathrm{~N}_{3} \mathrm{~S}$ & 2.00 & 2.33 & 339.20 & Fungicide \\
\hline Thifensulfuron-methyl & $79277-27-3$ & 387.39 & $\mathrm{C}_{12} \mathrm{H}_{13} \mathrm{~N}_{5} \mathrm{O}_{6} \mathrm{~S}_{2}$ & 1.27 & -1.64 & 170.50 & Herbicide \\
\hline Thiophanate-methyl & $23564-05-8$ & 342.39 & $\mathrm{C}_{12} \mathrm{H}_{14} \mathrm{~N}_{4} \mathrm{O}_{4} \mathrm{~S}_{2}$ & 1.10 & 2.36 & 438.90 & Fungicide \\
\hline
\end{tabular}


Table S2. Passive sampler uptake rate constants $(1 / h)$ for the different PAHs in the set-ups with and without dissolved organic carbon (DOC). These were used to calculate the time required for $95 \%$ equilibrium (h). Note that for benzo(a)pyrene in the saline medium set-up the uptake remained in the linear phase and no rate constant could be calculated (nc). For benzo(a)pyrene in the DOC set-up the time for $95 \%$ equilibrium was extrapolated.

\begin{tabular}{|l|c|c|c|c|}
\hline & \multicolumn{2}{|c|}{ Uptake rate constant (1/h) } & \multicolumn{2}{|c|}{$\begin{array}{c}\text { Time for 95\% } \\
\text { equilibrium (h) }\end{array}$} \\
& Medium & $\begin{array}{c}\text { Medium plus } \\
\text { DOC }\end{array}$ & Medium & $\begin{array}{c}\text { Medium } \\
\text { plus DOC }\end{array}$ \\
\hline Acenaphthene & 0.230 & 0.192 & 13 & 16 \\
Phenanthrene & 0.088 & 0.089 & 34 & 34 \\
Fluoranthene & 0.031 & 0.041 & 97 & 73 \\
Benzo(a)pyrene & $\mathrm{nc}$ & 0.016 & $\mathrm{nc}$ & 190 \\
\hline
\end{tabular}


Table S3. Equilibrium passive sampler concentrations ( $\left.C_{P D M S \text {,equil., }} \mu \mathrm{g} / \mathrm{L}\right)$ from fitting of the uptake curves together with the calculated dissolved concentrations $\left(C_{\text {free,calculated, }} \mu \mathrm{g} / \mathrm{L}\right)$ for the set-ups with and without dissolved organic carbon (DOC).

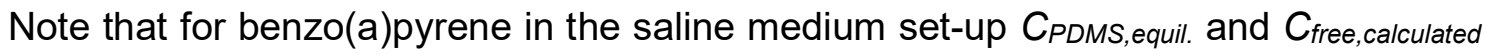
could not be calculated (nc).

\begin{tabular}{|c|c|c|c|c|c|c|}
\hline & \multirow[b]{2}{*}{$\begin{array}{c}K_{P D M S / \text { free }}{ }^{*} \\
\mathrm{~L} / \mathrm{L}\end{array}$} & \multicolumn{2}{|c|}{ Saline set-up } & \multicolumn{2}{|c|}{ DOC set-up } & \multirow[b]{2}{*}{$\begin{array}{c}C_{\text {free, measured }} \\
\mu \mathrm{g} / \mathrm{L}\end{array}$} \\
\hline & & $\begin{array}{c}C_{P D M S \text {,equil. }} \\
\mu \mathrm{g} / \mathrm{L}\end{array}$ & $\begin{array}{c}C_{\text {free,calculated }} \\
\mu \mathrm{g} / \mathrm{L}\end{array}$ & $\begin{array}{c}C_{P D M S, \text { equil. }} \\
\mu \mathrm{g} / \mathrm{L}\end{array}$ & $\begin{array}{c}C_{\text {free,calculated }} \\
\mu \mathrm{g} / \mathrm{L}\end{array}$ & \\
\hline Acenaphthene & 5994 & 1023000 & 170.7 & 1048000 & 174.8 & 161.0 \\
\hline Phenanthrene & 12720 & 167800 & 13.2 & 164100 & 12.9 & 12.4 \\
\hline Fluoranthene & 39940 & 2988000 & 74.8 & 2803000 & 70.2 & 80.0 \\
\hline Benzo(a)pyrene & 193445 & $\mathrm{nc}$ & $\mathrm{nc}$ & 49400 & 0.26 & 0.08 \\
\hline
\end{tabular}

* Taken from Smith et al (2015). 
Table S4. Mean $(n=2)$ passive sampler concentrations ( $\mathrm{ng} / \mathrm{kg}$ PDMS) for the WWTP inflow and outflow samples, as well as the receiving Saar river. PDMS sheets with thicknesses of 76 and $203 \mu \mathrm{m}$ were deployed in parallel for 4 (inflow and outflow) or 3 (Saar river) deployment periods. The laboratory determined equilibrium partitioning ratios (L/kg) are also shown in brackets after the compound names.

\begin{tabular}{|c|c|c|c|c|c|c|c|c|c|c|c|c|c|c|c|c|c|c|c|c|c|c|}
\hline $\begin{array}{l}\text { Concentration (nglkg PDMS) } \\
\text { Samming dates }\end{array}$ & 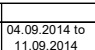 & 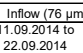 & 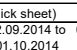 & 102010 & $\begin{array}{l}04.092 .21410 \\
11092010\end{array}$ & 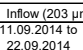 & 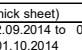 & 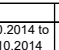 & $\begin{array}{l}04.0929014 \text { to } \\
11092014\end{array}$ & 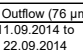 & 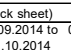 & $\begin{array}{ll}01.10 .201410 \\
101020210\end{array}$ & $\begin{array}{l}04.092 .21410 \\
11092010\end{array}$ & 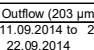 & 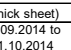 & 71.10.2014 to & 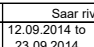 & 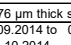 & $\begin{array}{l}\text { ett) } \\
10.2014 \text { to }\end{array}$ & 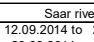 & 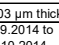 & 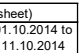 \\
\hline Carbendazim $(1.40 \mathrm{LLKg})$ & \begin{tabular}{|l|}
11.03 .2014 \\
78 \\
\end{tabular} & 的.2014 116 & 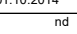 & (0.2014 & $\begin{array}{ll}96 \\
01.092 .214\end{array}$ & 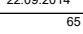 & 70 & 1028 & 636 & 22.092:2014 430 & $\frac{214}{691}$ & 10.10.2014 338 & \begin{tabular}{|l|l|}
17.09 .2014 \\
341
\end{tabular} & 22.99 .2014 & 留10.2014 431 & 10.10.2014 & \begin{tabular}{|l|}
23.99 .2014 \\
98
\end{tabular} & $\frac{10.2014}{185}$ & & \begin{tabular}{|l|}
23.09 .2014 \\
89
\end{tabular} & $\frac{10.2014}{218}$ & \\
\hline 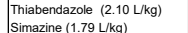 & 132 & 208 & 407 & & 163 & 125 & 645 & 147 & 1042 & 1024 & 2050 & 529 & 603 & 603 & 1236 & 1173 & 92 & 111 & 213 & 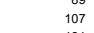 & 170 & \\
\hline $\mid \begin{array}{l}\text { Simazaine }(1.799 / / \mathrm{kg}) \\
\text { Atrazine }(2.07 \mathrm{Lkg})\end{array}$ & & $\begin{array}{l}\text { nd } \\
\text { nd }\end{array}$ & $\begin{array}{c}\text { nd } \\
\text { nd }\end{array}$ & nd & nd & $\begin{array}{c}\text { nd } \\
211\end{array}$ & nd & ${ }^{\text {nd }}$ & $\begin{array}{c}\text { nd } \\
864\end{array}$ & $\begin{array}{c}\text { nd } \\
10873 \\
10873\end{array}$ & $\begin{array}{r}80 \\
1718\end{array}$ & $\begin{array}{r}90 \\
486 \\
480\end{array}$ & $\begin{array}{r}83 \\
532-120\end{array}$ & 136 & $\begin{array}{r}104 \\
11159\end{array}$ & 1223 & 230 & $\begin{array}{r}175 \\
189\end{array}$ & ${ }_{1452}$ & 181 & 203 & \\
\hline 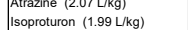 & $\begin{array}{c}\text { nd } \\
143\end{array}$ & $\begin{array}{l}\text { nd } \\
501\end{array}$ & $\begin{aligned} \text { nd } \\
400\end{aligned}$ & $\begin{array}{l}\text { nd } \\
250\end{array}$ & $\begin{array}{c}\text { nd } \\
132\end{array}$ & $\begin{array}{l}211 \\
474\end{array}$ & $\begin{array}{l}\text { nd } \\
416\end{array}$ & $\left.\begin{array}{c}\text { no } \\
592\end{array}\right]$ & $\begin{array}{l}864 \\
5122 \\
5\end{array}$ & $\begin{array}{l}18073 \\
7999 \\
79\end{array}$ & $\begin{array}{l}1718 \\
8171\end{array}$ & 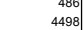 & $\begin{array}{r}522 \\
4085\end{array}$ & 7307 & $\begin{array}{l}1159 \\
5920\end{array}$ & 5382 & $\begin{array}{l}1995 \\
2019 \\
2\end{array}$ & $\begin{array}{l}1899 \\
2768\end{array}$ & 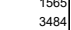 & $\begin{array}{l}1603 \\
1724\end{array}$ & $\begin{array}{l}1956 \\
3155\end{array}$ & \\
\hline $\begin{array}{l}\text { Dopron }(1.1 .14 \mathrm{Lkg}) \\
\text { Diron }\end{array}$ & 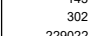 & 610 & 243 & $\begin{array}{r}208 \\
4083 \\
\end{array}$ & $\begin{array}{r}242 \\
240 \\
10309\end{array}-3$ & $\begin{aligned} 354 \\
3055\end{aligned}$ & $\begin{array}{r}176 \\
176\end{array}$ & 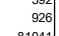 & 3915 & 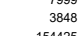 & $\begin{array}{r}3368 \\
3302\end{array}$ & $\begin{array}{l}4106 \\
8020 \\
8\end{array}$ & $\begin{array}{c}3455 \\
54520 \\
5\end{array}$ & 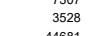 & $\begin{array}{l}2854 \\
28557 \\
6253\end{array}$ & $\begin{array}{c}48060 \\
48008 \\
9\end{array}$ & 2005 & $\begin{array}{l}1791 \\
57950 \\
5\end{array}$ & 26997. & 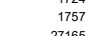 & 2007 & 246 \\
\hline 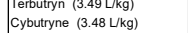 & 86 & 840 & $\begin{array}{c}339825 \\
118 \\
312\end{array}$ & $\begin{array}{l}1080034 \\
774\end{array}$ & $\begin{array}{l}183899 \\
82\end{array}$ & $\begin{array}{l}41055 \\
498 \\
498\end{array}-3$ & 224582 & $\begin{array}{c}81041 \\
1202\end{array}$ & $\begin{array}{l}257756 \\
551 \\
\begin{array}{r}2 \\
1\end{array}\end{array}$ & $\begin{array}{l}15425 \\
2142\end{array}$ & $\begin{array}{l}211938 \\
983\end{array}$ & 年2420 1760 & ${ }_{347}^{5522}$ & 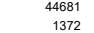 & ${ }_{6368}^{6357}$ & (200884 & $\begin{array}{c}62081 \\
1745 \\
6\end{array}$ & 52243 & 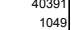 & 27165 & 48372 237 & \\
\hline Orysastrobin $(2.51 \mathrm{Lkg})$ & nd & nd & nd & . & nd & nd & nd & 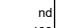 & nd & nd & 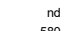 & 770 & wa & nd & nd & 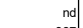 & 132 & 445 & 118 & 106 & & \\
\hline 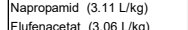 & nd & 157 & $\begin{array}{c}\text { nd } \\
\text { nd }\end{array}$ & not & nd & 91 & nd & 189 & 2017 & 1402 & 580 & 779 & 1818 & 1146 & 490 & $\begin{array}{r}937 \\
6611\end{array}$ & ${ }_{1726}^{926}$ & 1080 & 710 & 859 & 1490 & \\
\hline 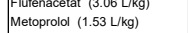 & $\begin{array}{l}\text { nd } \\
16515\end{array}$ & $\begin{array}{l}\text { nd } \\
12859 \\
\end{array}$ & $\begin{array}{c}\text { nd } \\
21933\end{array}$ & 吕 12243 & $\begin{array}{l}\text { nd } \\
9912\end{array}$ & 7969 & $\begin{array}{c}\text { nd } \\
\text { 12350 } \\
\text {. }\end{array}$ & $\begin{aligned} \text { nd } \\
14076\end{aligned}$ & $\begin{array}{l}390 \\
18614\end{array}$ & $\begin{aligned} 652 \\
13848\end{aligned}$ & $\begin{array}{l}9988 \\
15254\end{array}$ & $\begin{array}{r}54208 \\
14202\end{array}$ & $\begin{array}{r}2899 \\
8686\end{array}$ & $\begin{array}{r}650 \\
6577\end{array}$ & 7068 & . 60757 & $\begin{array}{l}11700 \\
13823\end{array}$ & 16946 & $\begin{array}{c}2586 \\
14135 \\
\end{array}$ & $\begin{array}{l}834 \\
7585\end{array}$ & $\begin{array}{l}3628 \\
9963 \\
963\end{array}$ & \\
\hline Cartamezapine $(1.62 \mathrm{Lkg})$ & 3301 & 3539 & 4476 & 3210 & 3711 & 2096 & 4263 & 5061 & 34569 & 22329 & 33027 & 24199 & $\begin{array}{l}18761 \\
18761\end{array}$ & 15931 & 21263 & 30102 & 7065 & 6 & 6785 & 4693 & 5388 & \\
\hline 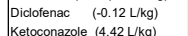 & $\begin{array}{l}945 \\
9608 \\
6808\end{array}$ & $\begin{array}{l}1305 \\
5141\end{array}$ & $\begin{array}{l}824 \\
6201 \\
6021\end{array}$ & $\begin{aligned} \text { nd } \\
5170\end{aligned}$ & $\begin{array}{l}1093 \\
3794\end{array}$ & $\begin{array}{l}1020 \\
3092 \\
302 \mathrm{C}\end{array}$ & $\begin{array}{r}319 \\
3421\end{array}$ & \begin{tabular}{r|r|r} 
nd & \\
8262 &
\end{tabular} & $\begin{array}{c}718 \\
4999 \\
4999\end{array}$ & $\begin{array}{l}{ }_{484}^{184} \\
4992\end{array}$ & $\begin{array}{l}1528 \\
3997\end{array}$ & $\begin{array}{r}3485 \\
345\end{array}$ & $\begin{array}{r}981 \\
2076\end{array}$ & $\begin{array}{l}1088 \\
2179\end{array}$ & $\begin{array}{l}1486 \\
1974\end{array}$ & $\begin{array}{r}108 \\
3094 \\
3.94\end{array}$ & $\begin{array}{l}539 \\
216\end{array}$ & $\begin{array}{l}514 \\
195 \\
195\end{array}$ & 830 & $\begin{array}{c}1088 \\
137 \\
137\end{array}$ & 582 & \\
\hline 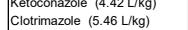 & $\begin{array}{l}68808 \\
168344\end{array}$ & $\begin{array}{l}51747 \\
17487 \\
\end{array}$ & $\begin{array}{r}6201 \\
334281\end{array}$ & 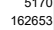 & 59417 & $\begin{array}{rl}3 & 3992 \\
43914\end{array}$ & $\begin{array}{l}3421 \\
73979 \\
73979\end{array}$ & $\begin{array}{r}8262 \\
154152\end{array}$ & $\begin{array}{r}449385 \\
14285 \\
\end{array}$ & $\begin{array}{r}4092 \\
1218889\end{array}$ & $\begin{array}{l}39004 \\
216045\end{array}$ & $\begin{array}{r}34857 \\
120157\end{array}$ & $\begin{array}{l}2016 \\
49260\end{array}$ & $\begin{array}{l}2179 \\
73969\end{array}$ & $\begin{array}{l}19 / 4 \\
67506\end{array}$ & $\begin{array}{c}39194 \\
19141\end{array}$ & $\begin{array}{r}216 \\
12385 \\
\end{array}$ & $\begin{array}{r}955 \\
9555\end{array}$ & 7484] & $\begin{array}{r}137 \\
6918\end{array}$ & $\begin{array}{l}146 \\
8987 \\
8987\end{array}$ & \\
\hline 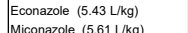 & $\begin{array}{r}7119 \\
17002\end{array}$ & 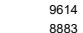 & $\begin{array}{l}6774 \\
9482\end{array}$ & $\begin{array}{l}{ }^{4986} 6 \\
8985\end{array}$ & $\begin{array}{l}5012 \\
6657\end{array}$ & ${ }_{4439}^{4539}$ & 5113 & $\begin{array}{r}7246 \\
13441\end{array}$ & $\begin{array}{l}2366 \\
1926 \\
296\end{array}$ & $\begin{array}{l}4953 \\
4439 \\
439\end{array}$ & $\begin{array}{l}{ }_{2832}^{2832} \\
{ }_{2498}\end{array}$ & 2980| 2711 & 10101 & $\begin{array}{l}2601 \\
2389\end{array}$ & 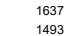 & 3554 & $\begin{array}{l}570 \\
230 \\
230\end{array}$ & $\begin{array}{l}379 \\
145 \\
145\end{array}$ & 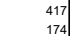 & $\begin{array}{l}390 \\
190 \\
100\end{array}$ & 4175 & 165 \\
\hline
\end{tabular}




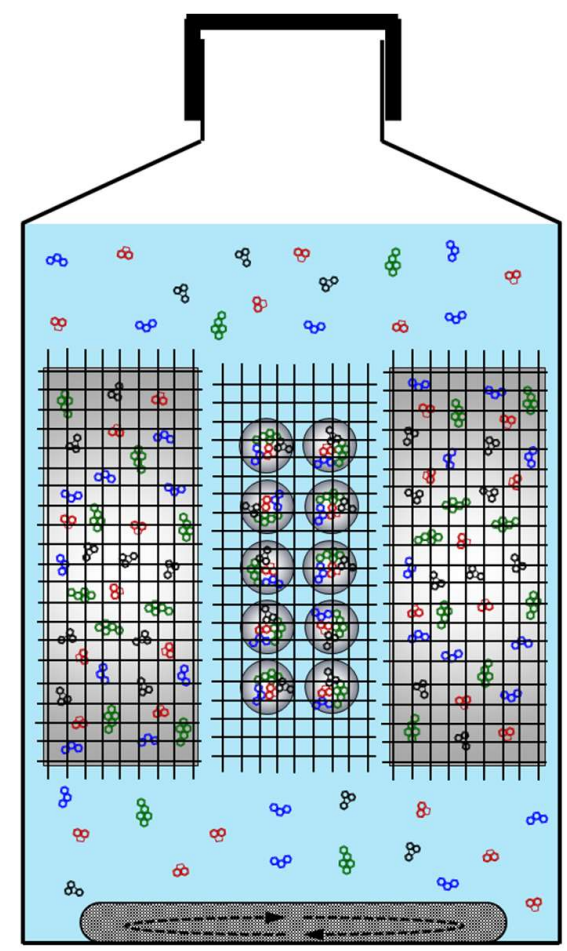

$2.1 \mathrm{~L}$ saline medium $\pm \mathrm{DOC}$

of 18 fluoranthene and benzo(a)pyrene

Passive sampler (exposed surface $56.55 \mathrm{~cm}^{2}$, volume $0.21 \mathrm{~cm}^{3}$ )

Passive dosing sheets (exposed surface $540 \mathrm{~cm}^{2}$, volume $10.96 \mathrm{~cm}^{3}$ )

Figure S1. Schematic of the experimental set-up. For maintaining constant dissolved concentrations, passive dosing was used. For this, PDMS sheets were loaded with PAHs and sandwiched in sets of two between stainless steel mesh. Three such passive dosers were added per bottle (only two are shown for clarity). As passive samplers, PDMS disks were sandwiched between two pieces of steel mesh and added to start the experiment. 


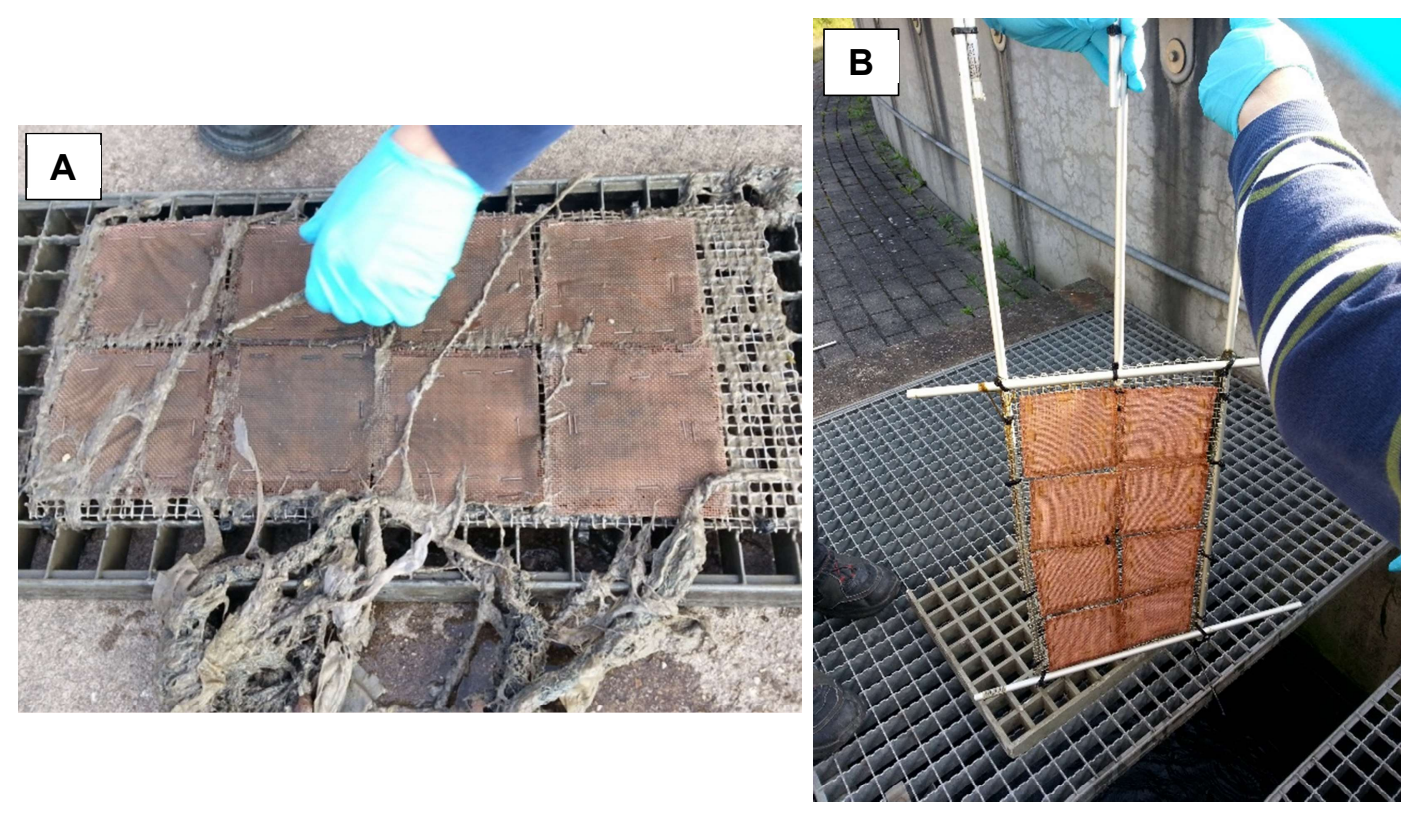

Figure S2. Photographs of the PDMS silicone sheets used as passive samplers recovered from the WWTP inflow $(A)$ and outflow $(B)$ after a 10-day deployment in September. 
(A) Medium

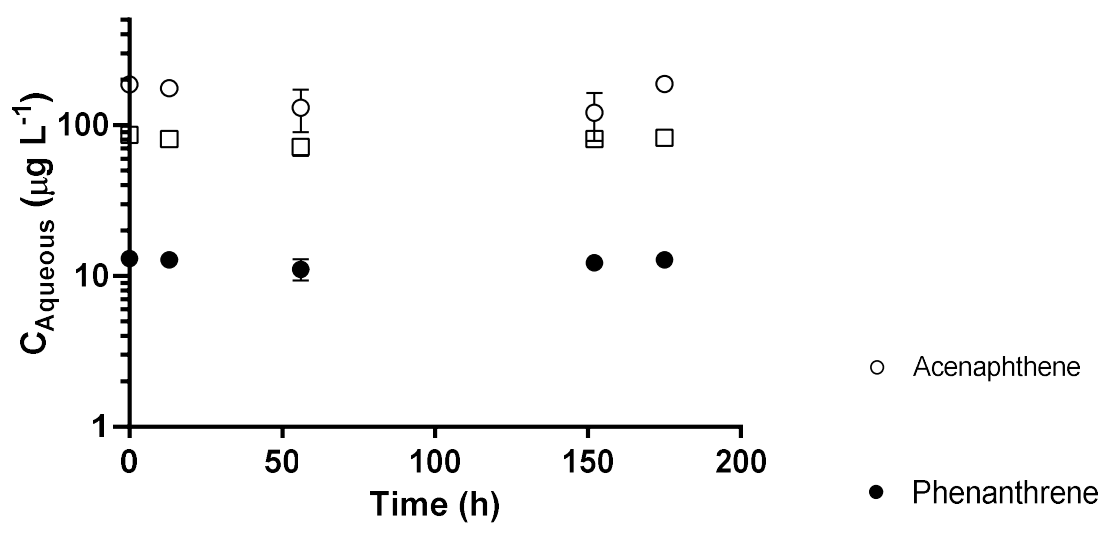

(B) Medium plus DOC

․ Fluoranthene

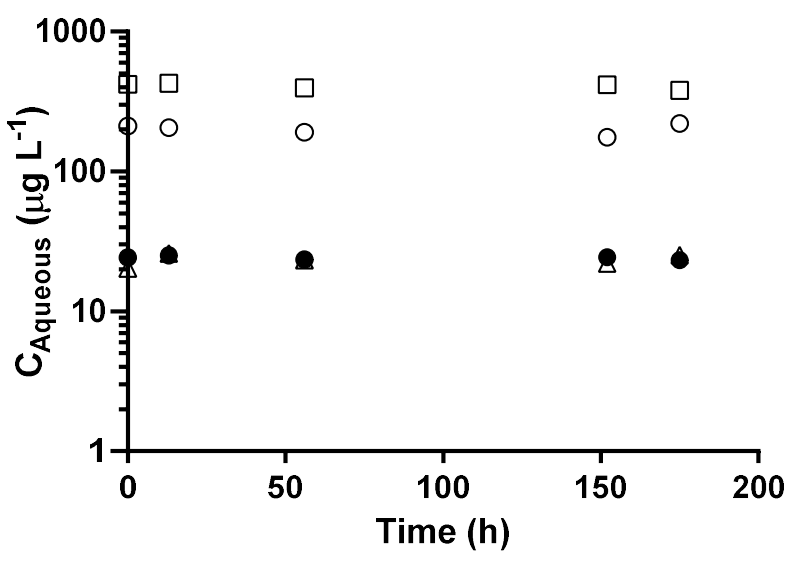

$\triangle$ Benzo(a)pyrene

Figure S3. Measured aqueous concentrations ( $\mu \mathrm{g} / \mathrm{L})$ in set-ups with and without 100 $\mathrm{mg} / \mathrm{L}$ dissolved organic carbon (DOC) $(n=3)$. Mean concentrations in saline medium were $160.8 \mu \mathrm{g} / \mathrm{L}$ (CV 23.5\%) for acenaphthene, $12.4 \mu \mathrm{g} / \mathrm{L}$ (CV 8.8\%) for phenanthrene and $80.6 \mu \mathrm{g} / \mathrm{L}(\mathrm{CV}$ 8.5\%) for fluoranthene. Benzo(a)pyrene concentrations were calculated to be $0.083 \mu \mathrm{g} / \mathrm{L}$ using the target $12 \%$ methanol saturation value and its seawater solubility. ${ }^{1}$ Mean values for medium plus DOC were $210.3 \mu \mathrm{g} / \mathrm{L}$ (CV 10.7\%) for acenaphthene, $24.2 \mu \mathrm{g} / \mathrm{L}$ (CV 5.1\%) for phenanthrene, 409.1 $\mu \mathrm{g} / \mathrm{L}$ (CV 5.5\%) for fluoranthene and $23.4 \mu \mathrm{g} / \mathrm{L}$ (CV 9.8\%) for benzo(a)pyrene. 


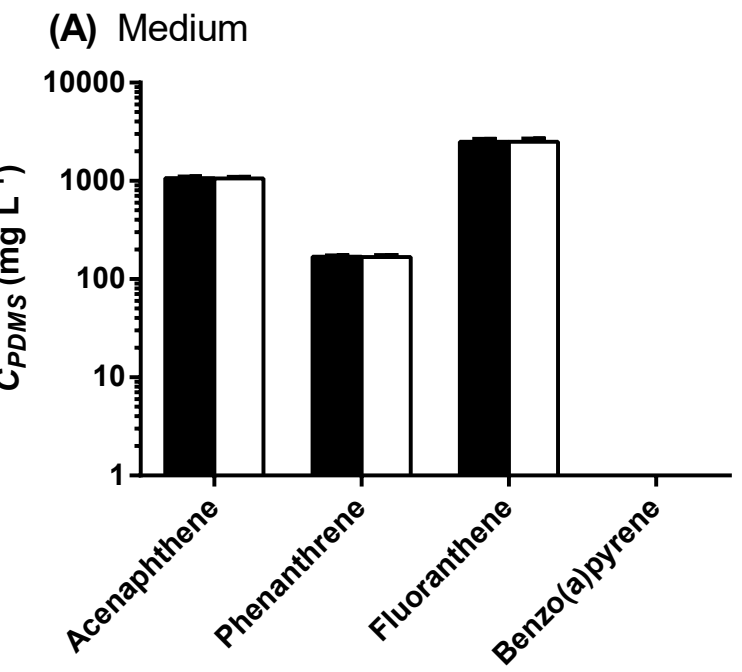

(B) Medium plus DOC

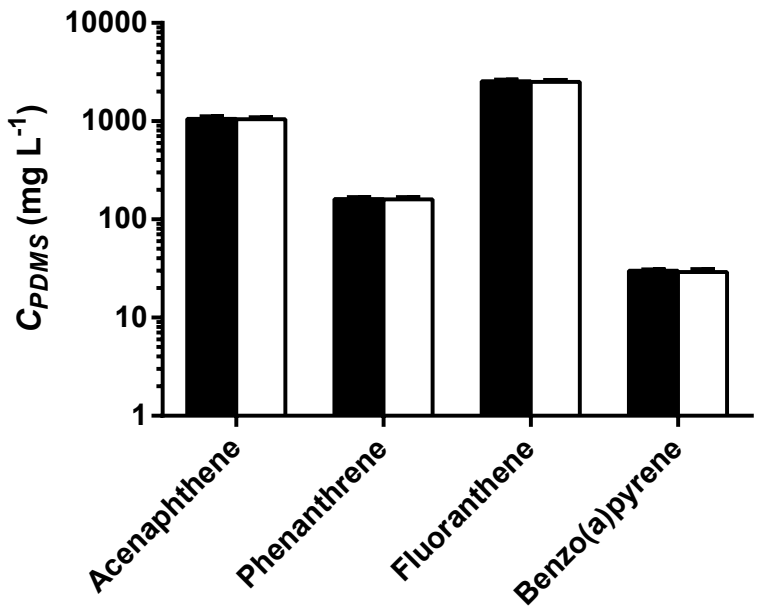

Analysis post passive dosing

Direct analysis

Figure S4. Comparison of the passive sampler concentrations from each set-up determined directly after sampling or after application as passive dosers in the Microtox® bioassay. Note that for the set-up with medium only, no benzo(a)pyrene concentrations could be determined due to the slow uptake kinetics. Columns represent the mean values and the error bars the standard deviation ( $n=3$ each). 
Linear Curvilinear Equilibrium

(A) Medium

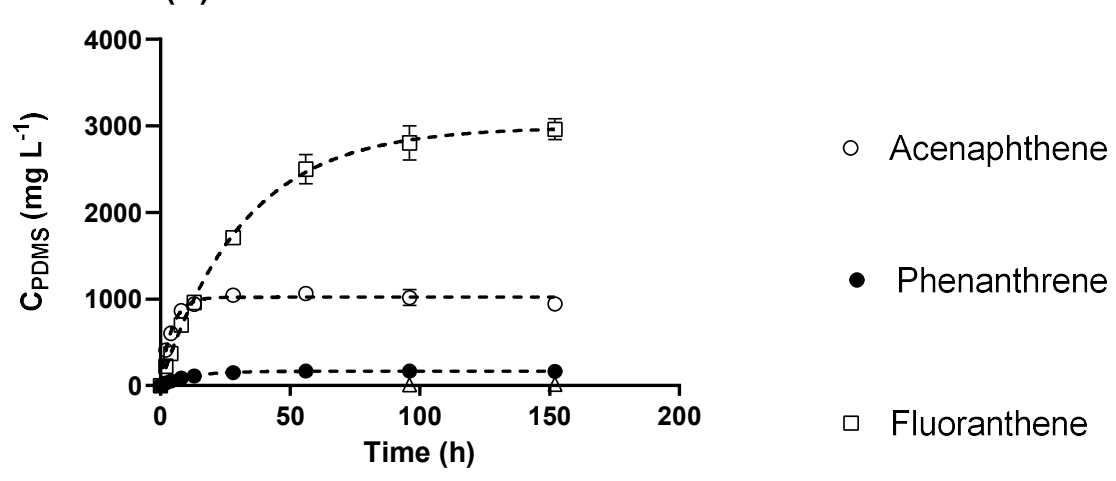

(B) Medium plus DOC

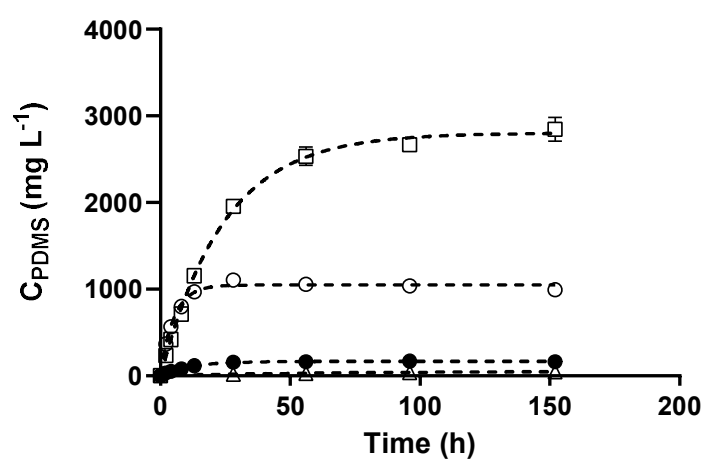

$\Delta$ Benzo(a)pyrene

(C) Benzo(a)pyrene

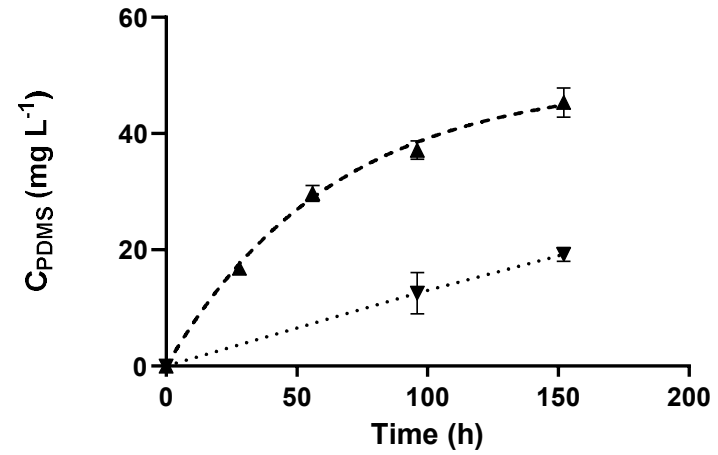

V Medium

- Medium plus DOC

Figure S5. Measured passive sampler concentrations $(\mu \mathrm{g} / \mathrm{L})$ of acenaphthene, phenanthrene, fluoranthene and benzo(a)pyrene in the set-ups containing medium with and without $100 \mathrm{mg} / \mathrm{L}$ dissolved organic carbon (DOC). The dashed lines show the best fit of the one-compartment model given by equation $\mathrm{S} 1$. The different uptake stages in the passive samplers are shown at the top. 


\section{Saline medium}

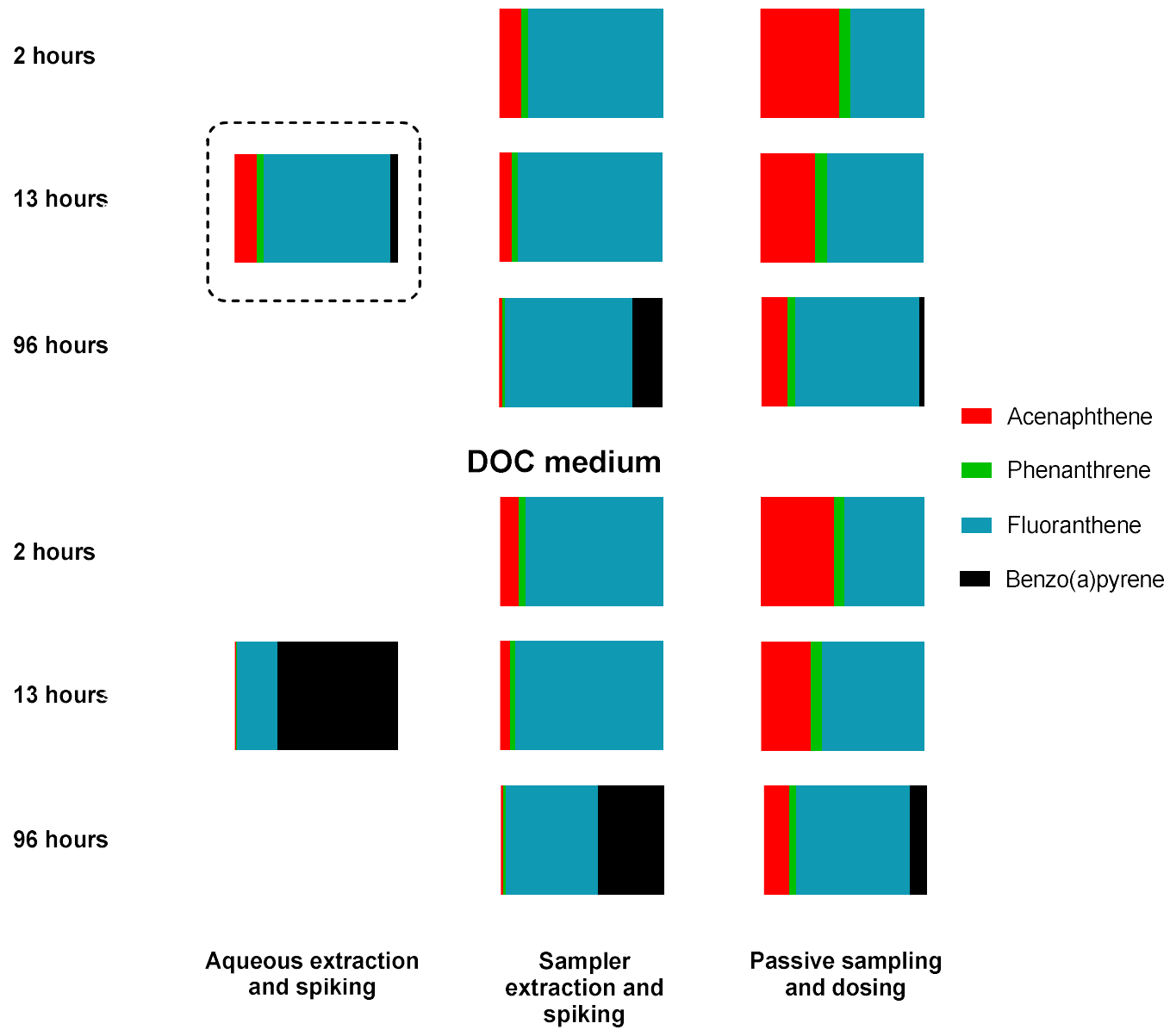

Figure S6. PAH chemical activity profiles for samples taken at 2, 13 and $96 \mathrm{~h}$ at the start of the Microtox bioassay for aqueous sample extraction and spiking, passive sampler extraction and spiking and passive sampling and dosing. For aqueous sample extraction and spiking, these profiles were unchanged since the dissolved concentrations were constant. Benzo(a)pyrene was only measured in the 96-day passive samplers due to its slower uptake. The activity profile of the extracted aqueous phase from the medium set-up is circled and represents the bioavailable profile for both set-ups. 


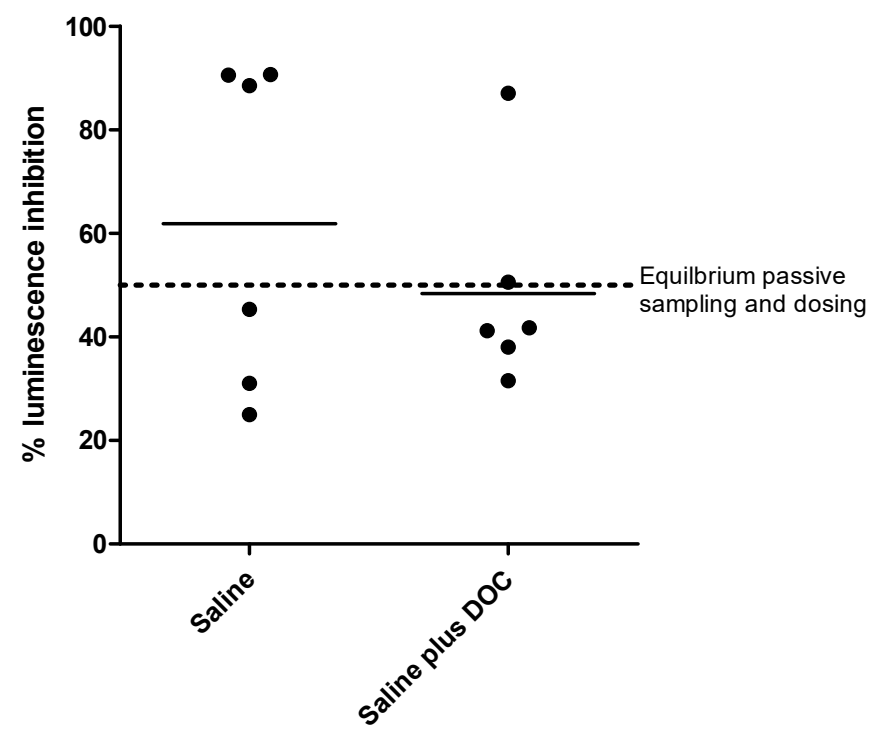

Figure S7. PAH mixture toxicities after extraction of $5 \mathrm{~mL}$ saline medium or saline medium plus DOC, exchange into $0.05 \mathrm{~mL}$ DMSO and spiking $1 \mu \mathrm{L}$ of this extract into the Microtox bioassay. Also shown is the measured mixture toxicity for equilibrium passive sampling and dosing as taken from the plateau in Figure 2. 
(A) Medium

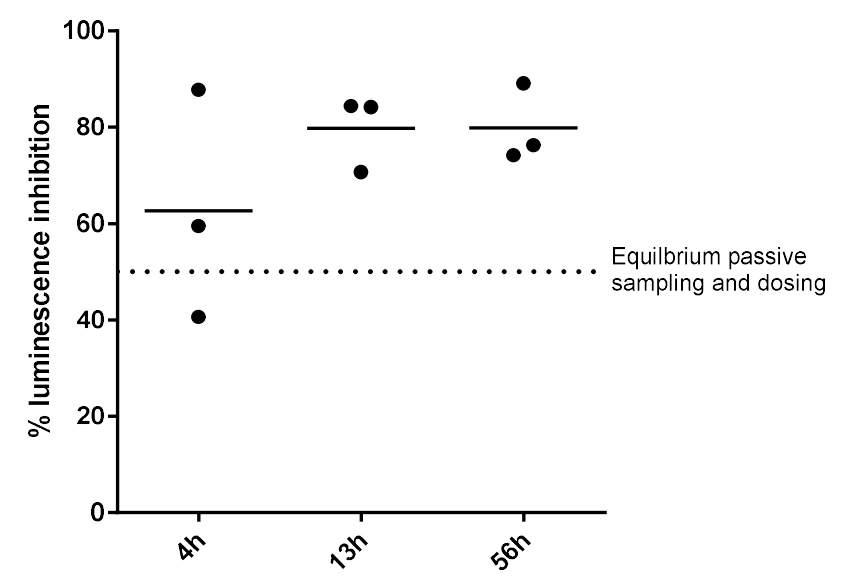

(B) Medium plus DOC

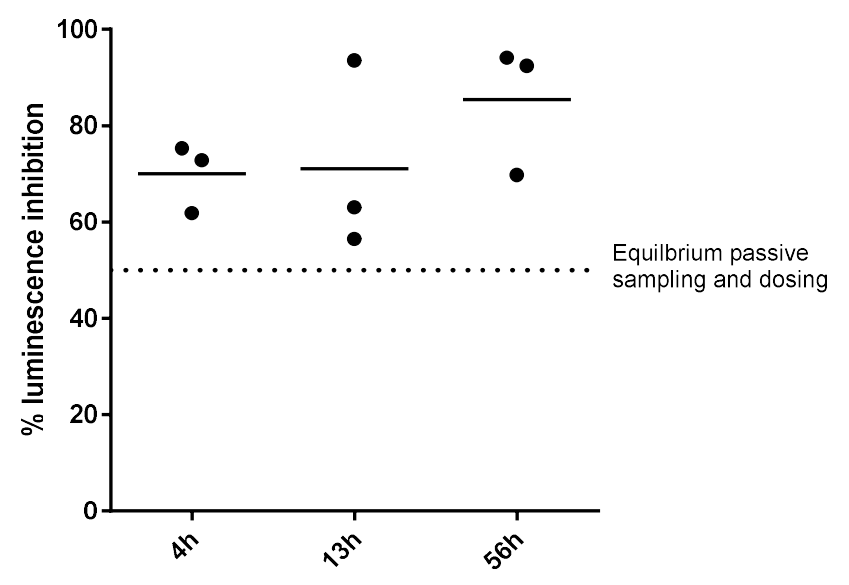

Figure S8. PAH mixture toxicities after extraction of passive samplers deployed for increasing lengths of time in $1 \mathrm{~mL}$ hexane, exchanging into $0.05 \mathrm{~mL}$ DMSO and spiking $1 \mu \mathrm{L}$ of this extract into the Microtox bioassay. Also shown is the measured mixture toxicity for equilibrium passive sampling and dosing as taken from the plateau in Figure 2. 


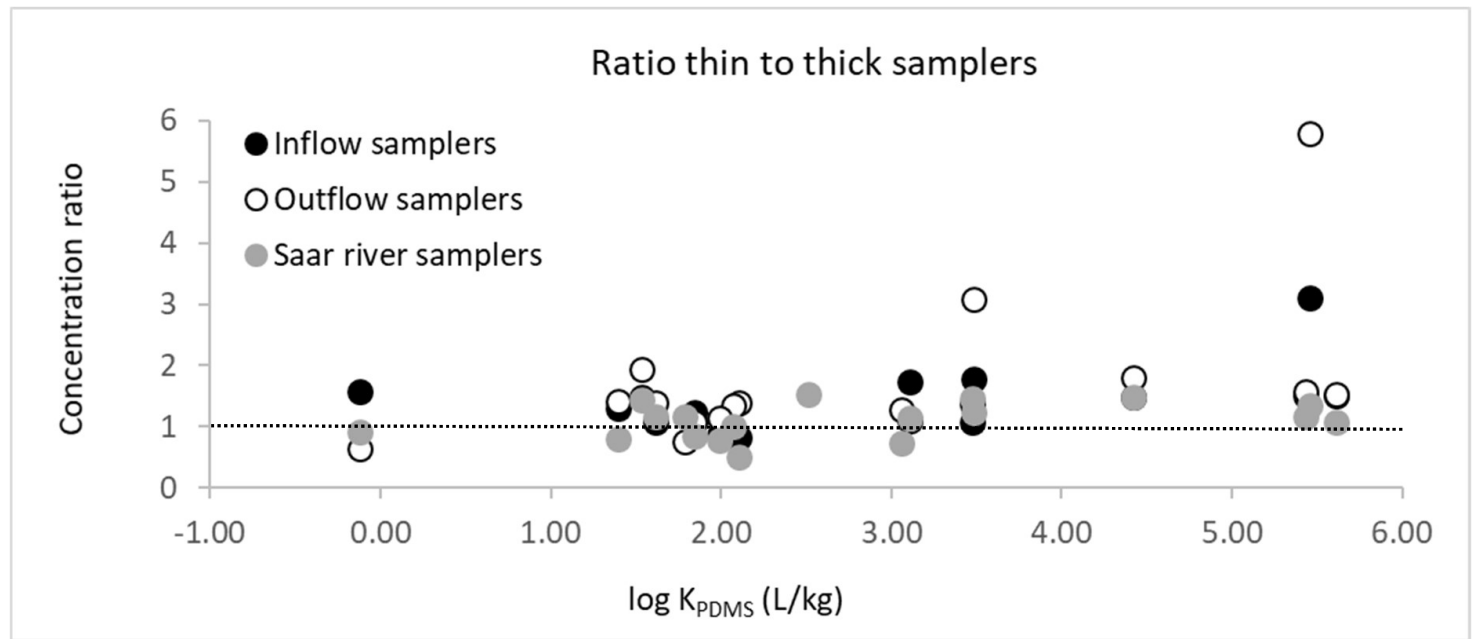

Figure S9. Ratio of the mean concentrations $(n=2)$ measured in the thin $(76 \mu \mathrm{m})$ and thick $(203 \mu \mathrm{m})$ passive samplers deployed in the WWTP inflow and outflow as well as Saar river. These are plotted against the mean sampler to water partitioning ratios (KPDMS, L/kg) of the detected compounds from Table S4. The dashed line serves as a visual guide for identical concentrations in both samplers represented by a ratio of 1 . 

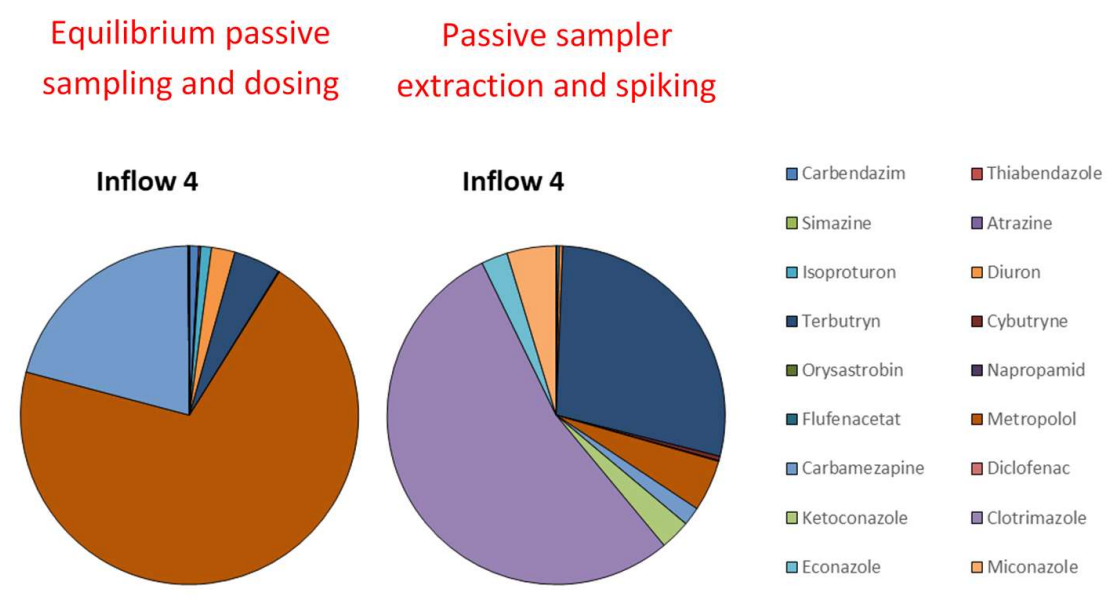

Outflow 4

Outflow 4
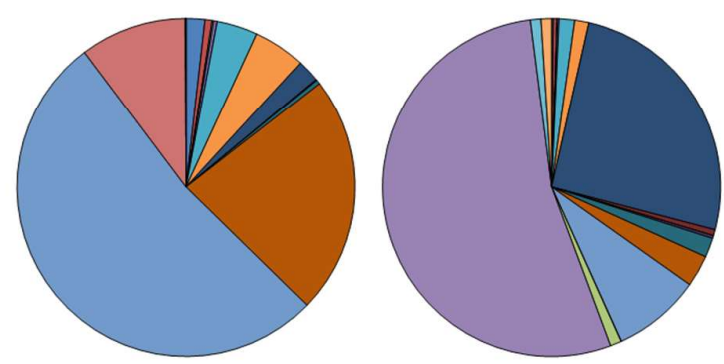

Saar river 4

Saar river 4
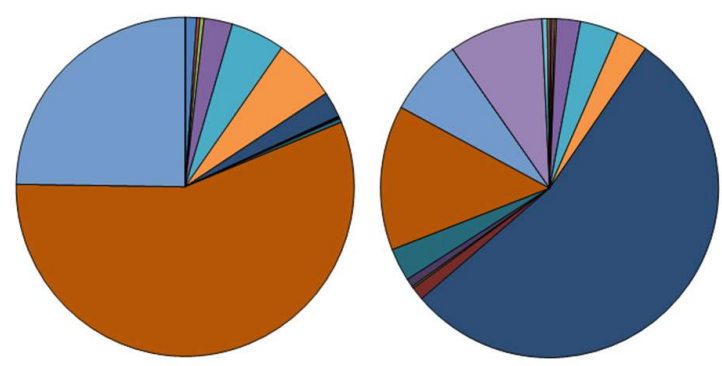

Figure S10. Initial mixture profiles of the environmental mixtures in the toxicity bioassays as set by equilibrium passive sampling and dosing (left column) and sampler extraction and spiking (right column). The equilibrium passive sampling and dosing values were calculated using the measured sampler concentrations and the appropriate compound sampler to water partitioning ratios (KPDMS, $\mathrm{L} / \mathrm{kg}$ ) from Table S4. These are exemplarily shown for one set of samples from the WWTP inflow and outflow as well as the Saar river. 


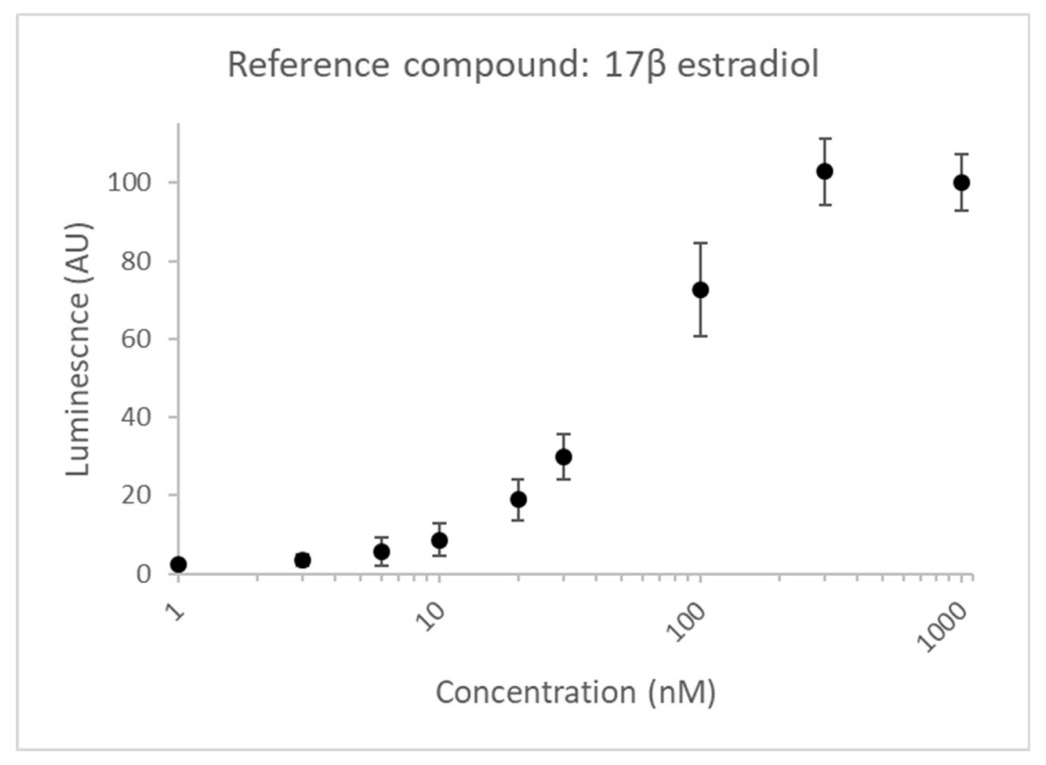

Figure S11. Concentration-response curve for the reference compounds $17 \beta$ estradiol in the ER Calux ${ }^{\circledR}$ bioassay. Each test plate included duplicate concentration-response curves, giving a total of 12 curves. Symbols show the mean and the error bars the standard deviation. 
(A)

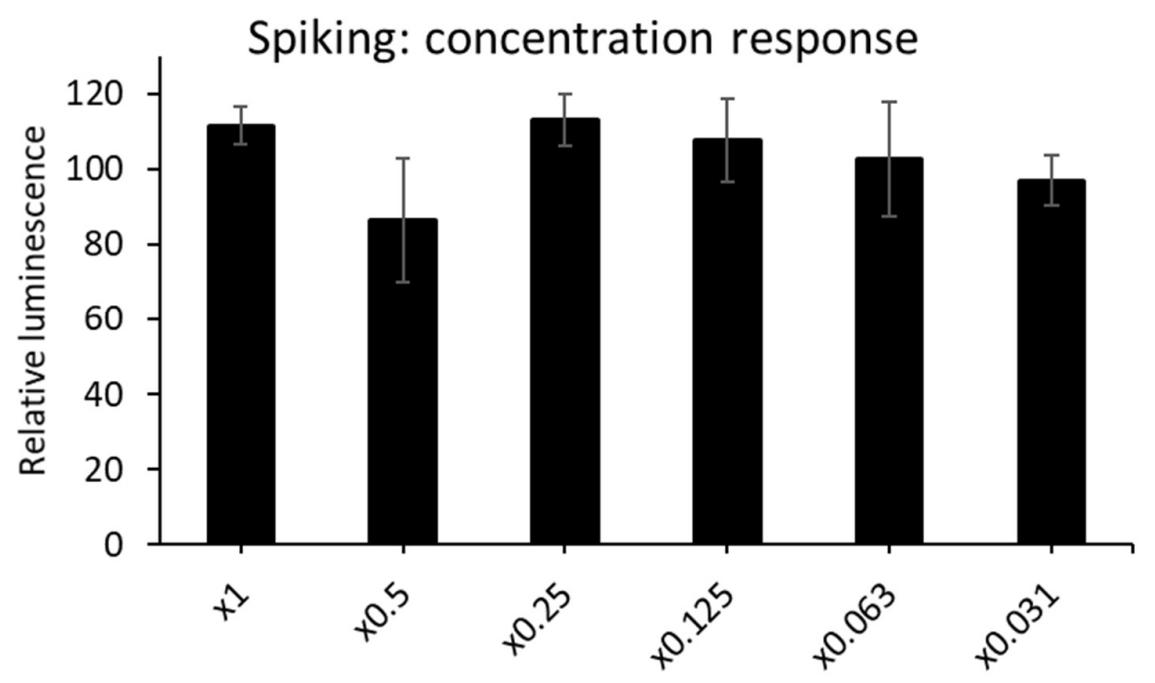

(B)

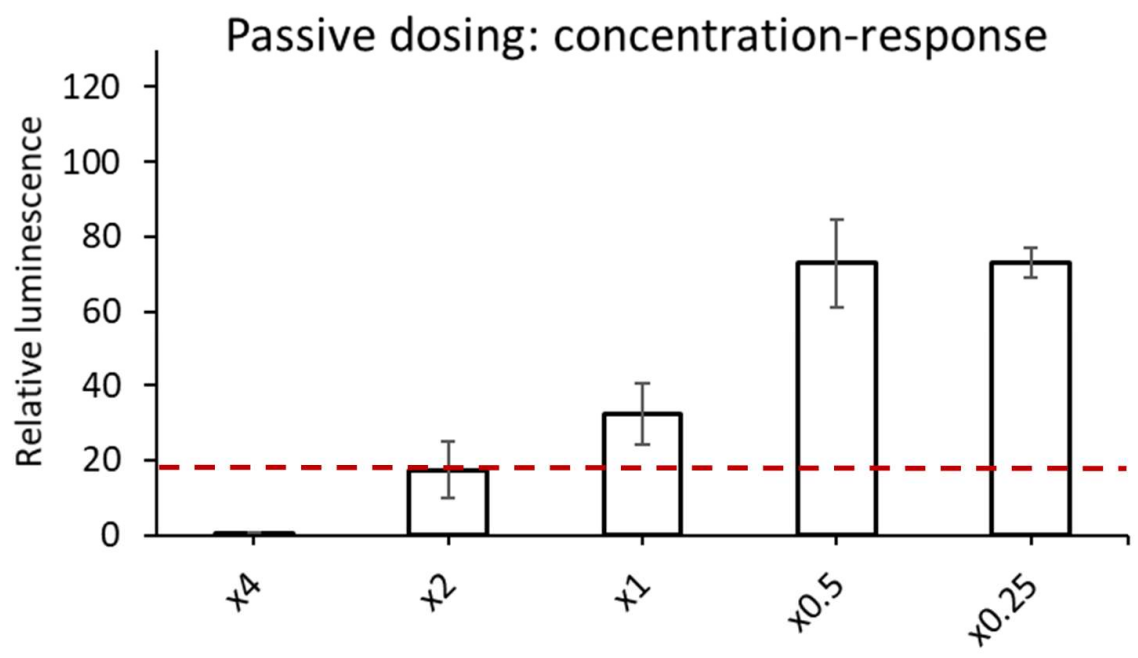

Figure S12. Calux bioassay concentration-response curve for the different levels of the spiked extracts (A) and passively dosed samples (B) from the inflow passive samplers recovered after the $3^{\text {rd }}$ deployment. Bars show the mean and the error bars the standard deviation $(n=3)$. The dashed red line shows the bioassay response measured for the field blank passive dosing disks (relative luminescence 18.9 \pm 5.7 standard deviation). 
References:

1. Reichenberg, F.; Smedes, F.; Jonsson, J. A.; Mayer, P., Determining the chemical activity of hydrophobic organic compounds in soil using polymer coated vials. Chemistry Central Journal 2008, 2, 10.

2. Rojo-Nieto, E.; Smith, K. E. C.; Perales, J. A.; Mayer, P., Recreating the seawater mixture composition of HOCs in toxicity tests with Artemia franciscana by passive dosing. Aquatic Toxicology 2012, 120-121, 27-34. 